\title{
Passer d'un modèle de fonctionnement associatif à un modèle institutionnel : une analyse des transformations de l'organisation du travail
}

From an associative to an institutional way of functioning: an analysis of the changes in work organization

Pasar de un modelo de funcionamiento asociativo a un modelo institucional : un análisis de las transformaciones de la organización del trabajo

Lysianne Rochat, Viviane Gonik et Brigitta Danuser

\section{(2) OpenEdition \\ Journals}

Édition électronique

URL : https://journals.openedition.org/pistes/1791

DOI : $10.4000 /$ pistes. 1791

ISSN : 1481-9384

Éditeur

Les Amis de PISTES

Édition imprimée

Date de publication : 1 novembre 2011

Référence électronique

Lysianne Rochat, Viviane Gonik et Brigitta Danuser, « Passer d'un modèle de fonctionnement associatif à un modèle institutionnel : une analyse des transformations de l'organisation du travail », Perspectives interdisciplinaires sur le travail et la santé [En ligne], 13-2 | 2011, mis en ligne le 08 octobre 2012, consulté le 21 septembre 2021. URL : http://journals.openedition.org/pistes/1791 ; DOI : https:// doi.org/10.4000/pistes.1791

Ce document a été généré automatiquement le 21 septembre 2021.

Pistes est mis à disposition selon les termes de la licence Creative Commons Attribution - Pas d'Utilisation Commerciale - Pas de Modification 4.0 International. 


\section{Passer d'un modèle de fonctionnement associatif à un modèle institutionnel : une analyse des transformations de l'organisation du travail}

From an associative to an institutional way of functioning: an analysis of the changes in work organization

Pasar de un modelo de funcionamiento asociativo a un modelo institucional : un análisis de las transformaciones de la organización del trabajo

Lysianne Rochat, Viviane Gonik et Brigitta Danuser

\section{Introduction}

1 Le monde de l'action humanitaire a connu des développements importants durant les dernières décennies. Sur les terrains d'interventions, les crises et les conflits se sont fortement complexifiés, nécessitant la participation de spécialistes de nombreux domaines (Cahill, 2006, p.10). Par ailleurs, les volumes financiers générés par les campagnes de dons et mis à disposition par les bailleurs de fonds ont considérablement augmenté. En corollaire de cette croissance financière, les exigences de contrôle et de traçabilité des fonds se sont renforcées. En lien avec ces éléments, le nombre de salariés dans les grandes organisations non gouvernementales a augmenté de manière exponentielle (Dauvin, Siméant, et C.A.H.I.E.R., 2002, p. 109). Une littérature spécifique sur les modalités d'évaluation des performances, le management et le « leadership » des organisations dites $\mathrm{du}$ "tiers secteur» a d'ailleurs vu le jour, comme l'illustre la naissance, en 1990, de la revue «Nonprofit Management and Leadership» (Young et 
Billis, 1990). Les pays bénéficiaires de l'aide ont également développé des exigences spécifiques envers les projets mis en œuvre par les ONG. Par des phénomènes de « socialisation des standards occidentaux» (Dauvin, 2004, p. 830), ces derniers attendent des acteurs internationaux un certain niveau de qualité des programmes.

2 Pour s'adapter à ces évolutions et répondre aux exigences d'efficacité auxquelles elles sont soumises, les organisations d'aide ont dû se transformer. Les grandes organisations ont ainsi connu durant les dernières décennies un mouvement de professionnalisation de leur structure, les conduisant à se rapprocher d'un modèle de fonctionnement que nous nommerons ici « institutionnel », à savoir formalisé et organisé. Nous employons ici le terme de professionnalisation dans l'appréciation qu'en font les acteurs du milieu humanitaire, à savoir en ce qu'il désigne « les restructurations internes auxquelles leurs organisations font face depuis la fin des années 1980 » (Le Naëlou, 2004, p. 775). Différents indicateurs de cette professionnalisation au sein des ONG peuvent être identifiés, notamment une plus forte division du travail, le développement de statuts spécifiques, la salarisation croissante des métiers de l'humanitaire ou encore le recours aux fonds publics (Dauvin et coll., 2002, p. 106).

3 Une conséquence également de cette évolution est l'entrée de nouveaux métiers sur la scène humanitaire. À côté des professions traditionnellement à l'origine des ONG (médecins, ingénieurs, juristes, etc.), la complexification et la diversification des tâches a rendu nécessaire de faire appel à des compétences professionnelles spécifiques dans des domaines tels que la communication, l'informatique ou la finance, pour ne citer que quelques exemples. Des connaissances et des pratiques spécifiques en matière de management des ONG se sont développées depuis la fin des années 1990 (Lewis, 2001, cité par Roberts, Jones III et Fröhling, 2005, p. 1849). Le métier de logisticien est apparu, lequel est enseigné dans des structures spécialisées (par exemple par l'association Bioforce en France) (Le Naëlou, 2004, p. 788). Des formations académiques spécialisées dans le domaine de l'humanitaire et de la coopération ont également vu le jour, avec le but affiché de former des professionnels spécialistes de l'humanitaire. On peut par exemple citer le PIAH en Suisse (Programme interdisciniplinaire en action humanitaire, 2011), ou encore les formations dispensées par le CIHC aux États-Unis (Center for international humanitarian cooperation, 2011).

\subsection{Problématique}

4 Passer d'un modèle associatif militant, au fonctionnement principalement informel, à un modèle institutionnel, dont la gestion est professionnalisée et le fonctionnement formalisé, entraine des transformations profondes de l'activité de travail. Nous pouvons penser que ceci a des effets importants sur les conditions de travail dans ces organisations, et par extension, sur la santé des personnes qui y travaillent.

5 Certains indices dans la littérature indiquent en effet que ces transformations structurelles ont un impact sur les conditions de travail et sur les employés des ONG dans lesquelles elles surviennent. Dans un article datant de 1983 déjà, Mirvis et Hackett attirent l'attention sur les risques liés à la professionnalisation du "tiers secteur »: ils voient dans la tendance à gérer ces organisations de la même manière que des entreprises une menace pour l'identité, la motivation et la satisfaction des employés (Mirvis et Hackett, 1983, p. 11). Plus récemment, Quéinnec (2007, p. 92) constate que l'institutionnalisation des ONG a des effets de durcissement de la situation de gestion et 
de diminution de la liberté d'action, lesquels sont «anxiogènes » pour les acteurs. Le Naëlou (2004, p. 777) observe également que les changements introduits dans le contexte de la professionnalisation sont liés à un malaise des employés, en ce qu'ils viennent questionner le sens du projet associatif que les personnes souhaitent donner à leur activité.

Cependant, peu d'études se sont attelées à documenter spécifiquement les résultats de ces transformations dans l'organisation concrète du travail et leurs effets sur le vécu et la santé des employés. Cet article présente une étude de cas des phénomènes concrets liés à la professionnalisation et de leurs impacts sur l'activité, au sein du siège d'une ONG humanitaire s'inscrivant dans le contexte décrit en introduction ${ }^{1}$. Nous verrons comment ces éléments se repèrent dans les modes d'organisation du travail actuels et comment ils se confrontent à la permanence du système associatif. Nous en questionnerons les effets sur le vécu des collaborateurs.

\section{Méthode}

\subsection{Terrain de recherche et population}

7 La population de cette étude est composée de tous les employés (salariés) du siège de l'ONG que nous avons étudiée. Cette dernière est une association à but non économique dans le sens de la loi suisse (art. 60 à 79 du Code civil) et est principalement active dans l'aide humanitaire d'urgence. Le nombre d'employés a légèrement évolué au cours des deux ans qu'a duré la récolte de données, passant de 132 en mars 2008 à 146 en mars 2010. La population est composée de $51 \%$ de femmes (chiffres de mars 2010) et la moyenne d'âge est de 40 ans $(S D=7.0)$. Cinquante pour cent des employés ont moins de 4 ans d'ancienneté (ancienneté max. : 17 ans), ce qui est un indicateur du turnover important observé par ailleurs. Soixante-quatre pour cent des employés du siège ont une expérience de travailleur expatrié avec cette ONG ou une autre organisation, pour une durée moyenne de 5 ans $(\mathrm{SD}=3.3)$.

8 On distingue deux entités formelles dans le fonctionnement de cette organisation: l'entité associative et l'entité exécutive. L'entité associative est représentée par les membres de l'association qui, pour la majorité, travaillent ou ont travaillé pour l'organisation, soit au siège ou sur le terrain ${ }^{2}$. L'entité exécutive de l'organisation est constituée du siège et du terrain. Le siège est responsable des décisions opérationnelles concrètes qui se prennent au quotidien. Il a une fonction de gestion et de soutien des activités du terrain et est chargé d'assurer les différentes tâches devant permettre l'atteinte des objectifs fixés lors de l'assemblée générale. Le terrain est le centre de toutes les préoccupations puisqu'il représente le lieu de la mission sociale de l'organisation et donc sa raison d'être (Rochat, Gonik et Danuser, 2010). Il est géré depuis le siège par le département des opérations, lequel occupe de ce fait une fonction centrale.

9 La récolte des données a eu lieu exclusivement au siège de l'organisation. Notre appréhension du terrain s'effectue donc par l'intermédiaire des représentations que nous en avons saisies depuis le siège. 


\subsection{Démarche méthodologique}

10 Cette recherche est née d'une intervention en santé au travail entamée à la demande de la direction du siège de l'ONG. Dans une première phase (phase diagnostique, voir tableau 1), nous avons réalisé une visite des locaux et organisé un focus group avec des représentants du personnel. Sur cette base, nous avons construit un questionnaire ${ }^{3}$ qui a été envoyé à tous les collaborateurs du siège de l'ONG (taux de réponse: 75/132 soit $56,8 \%$. Nous avons ensuite réalisé huit entretiens semi-directifs avec des personnes choisies au hasard parmi celles ayant indiqué être d'accord pour être recontactées dans le précédent questionnaire. La grille d'entretien comportait les thèmes de l'activité, de l'engagement, du choix professionnel, du stress et de la pression ressentie au travail. Les entretiens ont été intégralement retranscrits puis soumis à une analyse de contenu. Cette première phase nous a conduites à faire le constat de la présence d'un mal-être au travail important parmi les employés (voir point III.1) et a suggéré trois pistes d'analyses: le travail dans l'urgence en permanence, le fort engagement des employés envers leur organisation et leur activité et les transformations de la structure de l'organisation. Les deux premières pistes sont étudiées séparément (Rochat et coll., 2010), Rochat, Gonik et Danuser, en préparation).

11 Nous avons ensuite mis en place un projet de recherche pour mieux cerner l'organisation du travail et les aspects en lien avec la santé des travailleurs. La première auteure a effectué une période d'observation de 26 jours, passant une à trois journées dans chaque service et suivant en général ${ }^{4}$ une personne durant sa journée de travail en lui demandant de réaliser ses activités sans tenir compte de sa présence. Les questions suivantes nous ont guidées dans nos observations: quelle est l'activité de la personne; qu'est-ce qui détermine son activité présente; avec qui interagit-elle, dans quel but et de quelle manière (formelle/informelle); que se passe-t-il de manière générale autour de la personne? De nombreux échanges ont eu lieu durant la situation d'observation, sous forme de verbalisations et de discussions informelles sur les conditions et l'organisation du travail. Les notes prises sur le moment ont fait l'objet de relectures attentives puis ont été résumées et organisées selon la grille issue de l'analyse des précédents entretiens.

Une deuxième série d'entretiens semi-directifs a ensuite été réalisée par la première auteure afin d'approfondir certains éléments ayant émergé de la précédente analyse. La grille d'entretien comprenait les thèmes suivants : parcours professionnel ; engagement ; sens du travail; reconnaissance; limites à l'engagement; santé; stratégies de préservation de sa santé. Tous les collaborateurs de l'organisation ont été invités à manifester leur accord à participer à un entretien par courrier électronique. Douze personnes ont pu être rencontrées. Les entretiens ont été intégralement retranscrits et analysés à l'aide du logiciel Atlas Ti@ (version 6.1).

13 Les différentes phases de la recherche sont résumées dans le tableau 1 ci-dessous.

Tableau 1. Le déroulement de la recherche

\begin{tabular}{|l|l|l|l|l|l|}
\hline & Méthode & Date & Thématiques & Détails & Analyses \\
\hline
\end{tabular}




\begin{tabular}{|c|c|c|c|c|c|}
\hline \multirow[b]{2}{*}{$\begin{array}{l}\text { Phase } \\
\text { diagnostique }\end{array}$} & Questionnaire & $\begin{array}{l}\text { Mars } \\
2008\end{array}$ & $\begin{array}{l}\text { Nuisances au travail, } \\
\text { charge de travail, } \\
\text { conciliation avec la } \\
\text { vie privée, santé }\end{array}$ & $\begin{array}{l}\text { Taux de réponse : } \\
56,8 \%(75 / 132)\end{array}$ & $\begin{array}{l}\text { Statistiques } \\
\text { descriptives } \\
\text { Logiciel SPSS }\end{array}$ \\
\hline & $\begin{array}{l}\text { Entretiens } \\
\text { semi-directifs }\end{array}$ & $\begin{array}{l}\text { Juin - } \\
\text { août } \\
2008\end{array}$ & $\begin{array}{l}\text { Activité, engagement, } \\
\text { choix professionnel, } \\
\text { stress, pression } \\
\text { ressentie au travail }\end{array}$ & $\begin{array}{l}8 \text { entretiens, } \\
\text { sélection } \\
\text { aléatoire parmi } \\
\text { les personnes } \\
\text { ayant donné leur } \\
\text { accord dans le } \\
\text { questionnaire }\end{array}$ & $\begin{array}{l}\text { Analyse de } \\
\text { contenu }\end{array}$ \\
\hline \multirow[b]{2}{*}{$\begin{array}{l}\text { Phase de } \\
\text { recherche }\end{array}$} & $\begin{array}{l}\text { Analyses de } \\
\text { l'activité }\end{array}$ & $\begin{array}{l}\text { Sept. } \\
\text { - déc. } \\
2008\end{array}$ & $\begin{array}{l}\text { Activité, interactions, } \\
\text { environnement - } \\
\text { verbalisations et } \\
\text { discussions } \\
\text { informelles }\end{array}$ & $\begin{array}{l}26 \quad \text { jours } \\
\text { d'observation non } \\
\text { participante, } 1 \text { à } 3 \\
\text { jours dans chaque } \\
\text { service }\end{array}$ & $\begin{array}{l}\text { Notes papier- } \\
\text { crayon } \\
\text { Résumés et } \\
\text { analyses }\end{array}$ \\
\hline & $\begin{array}{l}\text { Entretiens } \\
\text { semi-directifs }\end{array}$ & $\begin{array}{l}\text { Juil. - } \\
\text { sept. } \\
2009\end{array}$ & $\begin{array}{l}\text { Parcours } \\
\text { professionnel, } \\
\text { engagement, sens du } \\
\text { travail, } \\
\text { reconnaissance, } \\
\text { limites à } \\
\text { l'engagement, santé, } \\
\text { stratégies de } \\
\text { préservation de sa } \\
\text { santé }\end{array}$ & $\begin{array}{lr}12 & \text { entretiens, } \\
\text { invitation } & \text { à } \\
\text { manifester } & \text { son } \\
\text { accord } & \text { par } \\
\text { courrier } & \\
\text { électronique } & \end{array}$ & $\begin{array}{l}\text { Analyse de } \\
\text { contenu } \\
\text { Logiciel Atlas } \\
\text { Ti }\end{array}$ \\
\hline
\end{tabular}

14 La méthodologie appliquée ici s'inscrit dans une démarche de triangulation méthodologique (Jick, 1979). Cette technique vise à combiner différentes méthodes dans l'étude d'un phénomène (Denzin, 1978), et permet ainsi de donner un cadre théorique à l'utilisation conjointe de méthodes quantitatives et qualitatives. L'intérêt est alors double : d'une part, l'utilisation de méthodes multiples permet de valider l'analyse dans le cas où les données vont dans le même sens, d'autre part, cette technique permet d'approfondir et d'affiner la théorie du chercheur lorsque celui-ci se trouve confronté à des données contradictoires. La triangulation méthodologique relève d'une stratégie inductive. Il s'agit d'une procédure d'allers-retours dynamiques entre les différentes phases de la recherche: le recueil de données, l'analyse et la conceptualisation (Apostolidis, 2006). Cette démarche se réclame ainsi d'une posture non positiviste et reconnaît au " chercheur-bricoleur » la capacité de mobiliser des outils propres à chaque situation pour étudier la complexité des phénomènes auxquels il est confronté (Denzin et Lincoln, 1998, cité par Apostolidis, 2006). Le but est ainsi d'étudier le plus de facettes possibles d'un phénomène donné (Flick, 1998, cité par Apostolidis, 2006) et de permettre l'émergence d'explications alternatives.

15 Concrètement, cette démarche s'est traduite dans notre recherche tant au niveau de la collecte de données que de l'analyse. Nous avons croisé et comparé les données récoltées à l'aide des différentes méthodes afin de renforcer la validité de notre recherche. La 
richesse de cette démarche réside dans le fait que chaque type de données vient éclairer des questions laissées ouvertes par d'autres types de données. Nous avons par exemple pu interpréter les données issues du questionnaire à la lumière des observations et des entretiens, ce qui a enrichi notre compréhension de certains phénomènes pointés par le questionnaire. Parallèlement, le questionnaire a permis de valider au niveau collectif certains aspects observés au niveau individuel. Cette démarche a ainsi permis d'assurer une compréhension large du champ de recherche et de renforcer le pouvoir de l'interprétation. Pour ces différentes raisons, cette démarche de triangulation s'est avérée particulièrement pertinente pour notre objet d'étude.

\section{Résultats}

Après une description rapide des indices de mal-être au travail, nous proposerons une présentation générale de l'organisation. Nous décrirons ensuite de manière concrète les modifications amenées dans cette structure dans le contexte de sa professionnalisation, ainsi que les aspects illustrant la persistance du fonctionnement associatif. Nous relèverons les effets de ces deux mouvements sur l'organisation du travail, sur l'activité quotidienne des employés et sur leur perception de leur travail. Le tableau 2, à la fin du point 3 , offre une vue synthétique des résultats.

\subsection{Indices de mal-être au travail}

Différents indices de mal-être au travail ont été observés dans l'organisation. De nombreux cas de ce qui est communément appelé « burnout » nous ont été rapportés. Par burnout, les employés entendaient le fait de craquer subitement, en raison de la pression subie au travail. Nous avons d'ailleurs observé que ce terme est d'usage courant dans l'organisation. Plusieurs personnes ont rapporté avoir été elles-mêmes victimes d'épuisement ou ont décrit des problématiques de santé qu'elles considéraient comme liées au travail. Les plaintes par rapport à la surcharge de travail étaient aussi nombreuses. De plus, la quasi-totalité des personnes rencontrées nous ont dit avoir été confrontées à des collègues proches quittant subitement pour cause d'épuisement, comme l'illustre l'extrait suivant :

Interviewé : "J'ai jamais vu autant de burnout [qu'ici], ça m'a fait halluciner.

Intervieweur : Par rapport à votre expérience professionnelle?

Interviewé: Oui, alors peut-être qu'avant je ne les ai juste pas remarqués, mais en fait avant je pensais que le burnout c'était un peu... que tu n'as plus tellement envie et puis un peu psychologique, et en fait non pas du tout [...]. [Maintenant] je comprends comment on peut arriver à ce stade, c'est-à-dire effectivement d'aller tellement trop loin et d'essayer de se dépasser ou de prendre beaucoup plus de travail qu'à un moment donné on arrive à un épuisement et on n'arrive pas à le gérer. [...]

Intervieweur: Vous avez vu beaucoup de monde comme ça?

Interviewé: Oui j'ai vu beaucoup de monde et dernièrement j'ai vu beaucoup de monde dans notre département, en fait [...] l'année dernière il y a eu cinq [burnout] [...].

Intervieweur: Votre département, ça représente une vingtaine de personnes quelque chose comme ça? [...]

Interviewé : Oui à peu près vingt à vingt-cinq personnes [...] je trouve ça vraiment énorme, et puis des fois je me suis dit que j'étais pas loin.» (14/8/34) 
Une autre personne remarque la détérioration progressive de son état général depuis son arrivée au siège et dit être arrivée à un point qui l'oblige à envisager de diminuer son activité au sein de cette organisation :

Interviewé: "Aujourd'hui physiquement je peux plus. [...] En deux ans j'ai pris dix kilos parce que je compense assez par la bouffe. [...] Il m'arrive assez souvent dans la semaine de m'endormir épuisée à neuf heures et demie, dix heures sur l'article que je suis en train de lire, et puis de me réveiller en sursaut à trois heures du matin et de ne plus pouvoir dormir parce que je cogite. Ça m'arrive [d'être] d'excellente mauvaise humeur à la maison et d'envoyer tout le monde péter parce que je suis vraiment fatiguée, et ça c'est un peu embêtant, ça m'arrive de pleurer en me disant, je peux plus... Et puis j'essaie d'être extrêmement organisée pour gagner mon temps, et là, en ce moment j'arrive même plus. Hier j'ai raté mon train à cinq minutes près. [...] En ce moment je suis à un tel point que même en écrivant tout sur mon agenda ben j'arrive même plus à le suivre. [...] J'oublie des choses, des fois je suis en train de me concentrer en me disant: t'étais là, t'as fait ça... et après où t'es allée où t'as mis le truc? Je ne sais plus. Ça m'arrive d'appeler les gens en leur disant on avait parlé de ça, [...] je me rappelle plus si on avait fini, sur quoi... Oui oui, ça devient des trucs tu te dis... » (13/12/2)

19 Par ailleurs, nous avons mis en évidence que la population de l'étude a une perception de sa santé moins bonne que la population suisse active, bien que cette dernière ait une moyenne d'âge plus élevée. À la question " comment est votre santé en général ? ", les employés de l'ONG étudiée sont $79,8 \%$ à répondre bonne ou très bonne, contre $93 \%$ pour la population suisse (Krieger et Graf, 2009, p. 33). De plus, au test de santé total de Langner et Amiel (Amiel, 1986), environ un tiers des répondants ont signalé des problèmes de mémoire, des troubles du sommeil, ainsi qu'une forte irritabilité et de la nervosité.

Ce mal-être se traduit par un rapide turnover. La durée moyenne d'occupation d'un poste est évaluée à trois ans par le département des ressources humaines. Pour tenter de limiter le fort turnover, l'organisation a mis en place un contrat moral au travers duquel les employés, lors de leur arrivée au siège, s'engagent à rester pour une durée minimum à leur poste (en général trois ans). Lors de nombreux entretiens et discussions, la problématique du turnover était mise en lien avec celle de l'épuisement, comme l'illustre l'extrait suivant:

Interviewé: "Il y a un turnover massif dans cette boite, moi je suis là depuis quatre ans je suis un dinosaure! [...] Les gens sont épuisés et s'en vont, et il y a des gens qui sont partis, mais dans des états... » (13/19/13)

21 L'absentéisme est également une préoccupation importante de la direction. Il n'a malheureusement pas été possible de le chiffrer concrètement; néanmoins, en plus du rapide turnover et des plaintes du personnel, c'est ce qui a conduit la direction de l'organisation à demander un diagnostic sur les conditions de travail.

22 Ces éléments, pris dans leur ensemble, tendent à montrer la présence d'un mal-être au travail non négligeable.

\subsection{L'exercice du pouvoir dans l'organisation}

23 À son origine, cette organisation était une petite association portée par un nombre restreint de personnes fortement engagées. Comme nous l'avons relevé en introduction, la croissance qu'a connu cette ONG et l'évolution du contexte de l'humanitaire ont amené de profondes transformations dans les modes de fonctionnement du siège. Plusieurs « anciens » ont évoqué avec nostalgie l'époque où les décisions se prenaient autour d'une 
table à l'issue de discussions impliquant tous les membres de l'association. Avec la forte augmentation du nombre de salariés du siège, ce fonctionnement n'est évidemment plus possible. Au cours des dernières années, les entités associatives et exécutives se sont vu de plus en plus séparées, chacune se voyant attribuer un mode de fonctionnement propre. Ceci s'est en réalité surtout traduit par une distanciation du mode de fonctionnement de l'exécutif (à savoir du siège) d'un mode de fonctionnement purement associatif.

24 La situation de gouvernance s'est fortement complexifiée et nous avons relevé des référentiels de pouvoir multiples. Du point de vue du siège, trois systèmes de références exerçant du pouvoir dans l'organisation ont été identifiés :

\subsubsection{Le système associatif}

25 Le pouvoir de l'entité associative s'exerce sur le personnel du siège de la manière suivante : les membres de l'association élisent les membres du conseil d'administration, lequel est chargé de faire appliquer la stratégie globale décidée par l'assemblée générale par l'intermédiaire du conseil de direction.

\subsubsection{Le système hiérarchique}

\subsubsection{Le système opérationnel ${ }^{5}$}

Le troisième système de référence de pouvoir dans l'organisation se réfère à la légitimité opérationnelle des différents acteurs du siège. Indépendamment de la place d'une personne dans la hiérarchie, sa proximité avec les activités du terrain lui confère un pouvoir de décision par rapport à de nombreux éléments, opérationnels et aussi opérationnels. Le pouvoir décisionnel est ainsi en grande partie concentré dans les mains du département des opérations. Ce dernier est d'ailleurs vu par tous comme «le département qui décide» et qui valide, voire contrôle ce que font les autres départements. La légitimité d'une fonction n'est ainsi pas définie en fonction de sa place dans la hiérarchie, mais en fonction de son degré d'opérationnalité. 
Cependant, de nombreux collaborateurs des départements techniques (non opérationnels donc non décisionnels) ont une expérience opérationnelle et donc aussi une expertise dans ce domaine-là. Afin de profiter de l'expertise et de l'expérience du plus grand nombre, la direction a mis en place un espace de débat pour les questions opérationnelles au siège où chacun est invité à donner son avis et à poser les questions qu'il souhaite. Néanmoins, si auparavant les décisions étaient prises à l'issue de ces séances par votation, la clarification du pouvoir de décision dans l'organisation a amené à la restriction du pouvoir de cette séance. Cette dernière a désormais une fonction uniquement consultative et non plus décisionnelle. La décision est prise par le comité de direction, dans lequel la direction des opérations joue un rôle important. La direction affiche ainsi clairement sa volonté de dissocier les différents niveaux décisionnels, et bien qu'elle encourage la prise de position et le partage des idées en maintenant des espaces de débat (ce qui tend à entretenir une logique associative), elle est désormais très présente pour encadrer et réguler ces échanges (ce qui témoigne d'une logique plutôt institutionnelle). Dans les faits, ceci est vécu par de nombreux employés comme un nonrespect de leur expertise, comme l'illustre l'extrait suivant avec le responsable d'une équipe technique :

Interviewé: " Il y a une espèce de frustration qui date depuis que tu es sur le terrain, tu fais des opérations au quotidien [...] mais tu n'as pas de responsabilités opérationnelles. Et depuis le début, depuis tes postes [sur le terrain] [...] et maintenant à mon poste, moi j'ai l'impression d'avoir digéré ces frustrations et de les avoir oubliées, mais [les personnes de mon équipe] sont encore en plein là-dedans.

Intervieweur : Tu n'as pas de pouvoir de décision?

Interviewé: Non, et eux non plus, c'est-à-dire qu'ils accompagnent les opérations, ils n'en conduisent pas mais ils les accompagnent, [...] ils donnent leur avis, ils recommandent, ils conseillent, mais ils n'ont pas de pouvoir de décision, le seul veto qu'on a ce sont sur des problèmes techniques assez... Et encore, l'opération a tout pouvoir. » (21/9/47)

Les éléments présentés ici mettent en évidence la cohabitation de référentiels de pouvoir multiples (pouvoir associatif, pouvoir de la direction, légitimité opérationnelle), ce qui crée une situation dans laquelle coexistent différentes chaînes de commandement, certaines formelles, d'autres plus informelles.

\subsection{Une codification du travail plus formelle}

31 Au cours des dernières années, de nombreux outils ont été introduits, tels que cahiers des charges, évaluations annuelles, règlements, processus, etc. Nous nous sommes intéressées à leur utilisation par les acteurs de l'organisation.

Un cahier des charges a été établi pour chaque collaborateur dans le but de clarifier les tâches et les responsabilités qui relèvent de chaque fonction et de formaliser le partage des tâches. Cependant, sa pertinence semble mise en doute par de nombreux employés. La plupart ont relevé qu'il est très sommaire et qu'il n'est pas tenu à jour. Il existe de plus une règle implicite qui veut que chacun s'investisse au-delà de ce qui y est indiqué. Quant au partage des tâches, bien qu'il ait été également formalisé, nous avons observé qu'au nom de l'urgence, il est fréquent que des personnes réalisent des tâches qui ne relèvent pas directement de leurs fonctions, ce qui peut créer des tensions.

La direction a également mis en place un système de fixation d'objectifs qui se ramifient par départements, services et finalement, pour chaque employé. L'atteinte de ces objectifs est évaluée dans un entretien annuel individuel entre l'employé et son supérieur 
direct. Ici également nous avons observé une forte ambiguïté dans l'appréciation de ce système par les employés. D'une part, ils relèvent que les évaluations sont purement formelles et ils ne leur attribuent pas d'effets sur leur parcours au sein de l'organisation. Mais d'autre part, au vu de leur fort engagement personnel dans leur activité, ces objectifs, qu'ils fixent en partie eux-mêmes, leur tiennent à cœur. Ils sont cependant le plus souvent si ambitieux qu'il est difficile de les atteindre. Plusieurs personnes ont dit ressentir une importante frustration et avoir le sentiment que leurs efforts ne suffiront jamais.

La formalisation des tâches a également nécessité la mise en place de règles et de processus pour structurer les rapports de travail. Bien que des règlements existent, de nombreux employés ont relevé que les règles formelles ne s'appliquent pas à tous de la même manière. De nombreux éléments sont laissés à la discrétion des chefs de service et certaines possibilités ne sont pas connues de tous. Il semble y avoir, dans certains départements du moins, l'idée que le volontariat et la motivation permettent de faire l'économie de ces règles justement. Ceci génère de forts sentiments d'inégalité :

Interviewé: "La direction a mis en place [...] une somme de procédures qui pour certaines étaient bienvenues, mais qui ne sont déjà plus respectées ou qui sont respectées parfois, pas toujours, selon les personnes. Donc le deux poids deux mesures, ce n'est juste pas possible. Ça n'a déjà plus de légitimité. [...] L'exception ne peut pas être acceptée dans ce genre de choses, elle fait tomber la procédure d'elle-même. Donc, il y a une façade qui apparemment est voilà, on produit beaucoup de [papiers], mais dans l'application c'est un peu n'importe quoi.» (17/18/13)

D'une manière générale, nous avons remarqué une perception relativement négative des aspects managériaux, lesquels sont les principaux vecteurs de la professionnalisation. Le fait de travailler pour une association suscite des attentes importantes chez les collaborateurs. Ce milieu est très souvent idéalisé comme faisant preuve d'une gestion " plus humaine » que les grandes entreprises, dans lequel le but est de travailler ensemble " pour la cause ». Cependant, en lien avec la croissance de la structure, la gestion est perçue comme devenant de plus en plus impersonnelle. La hiérarchie est parfois perçue comme n'étant pas suffisamment attentive à la souffrance des employés et peu soutenante dans les situations difficiles. Plusieurs personnes ont relevé n'avoir eu personne vers qui se tourner lors de périodes tendues, que ce soit en raison d'une charge de travail trop importante ou à cause de relations professionnelles conflictuelles. Dans un contexte de travail difficile et exposé à la souffrance d'autrui, habité de plus par un idéal d'engagement très fort, ce manque de soutien et le sentiment de subir les décisions managériales conduisent à un questionnement autour du sens du travail et génèrent un mal-être important, comme l'illustrent les deux extraits suivants.

Interviewé: "[L'organisation] grossit. Et l'adaptation de la structure à cette réalité rend le management plus impersonnel. [...] Ce dont je souffre aujourd'hui, c'est que je suis un pion dans une institution à qui il est demandé de faire un certain nombre de choses, au lieu de:je suis un membre ou une partie prenante d'une institution dans laquelle je connais la finalité de mon travail qui est bien plus large que ce que je fais, mais où je travaille pour le PROJET [de l'organisation]. Et aujourd'hui le mode de management, certainement dû au fait que c'est devenu plus grand et que donc tout le monde ne peut pas participer à toutes les décisions, oui bien sûr, mais on ne l'a jamais demandé, mais qu'on soit au courant de ce vers quoi on va et vers quoi nos braves dirigeants veulent nous mener, ça c'est obligatoire. Et je ne le retrouve pas. [...] On me dit ce que je dois faire, et je ferais ça [dans un supermarché] ou [ici], c'est la même chose. » (19/8/45)

Interviewé: "Ce genre de métier pour moi c'est un taureau qu'il faut prendre par les cornes, c'est tout, et j'ai fait en sorte de le prendre par les cornes pour pas qu'il ne m'attaque trop. [... 
] Mais par contre, finalement quand je m'attelais à tenir ce taureau, c'est venu par derrière! Intervieweur:Et ça justement c'est un peu toute cette organisation, ce type de fonctionnement?

Interviewé : Voilà. » (12/21/17)

\subsection{La valorisation croissante des formations universitaires}

Un aspect tenant également de cette professionnalisation est la valorisation croissante des diplômes et des formations universitaires. S'il semble qu'auparavant l'expérience et l'apprentissage en situation étaient avant tout valorisés pour accéder à un poste hiérarchique par exemple, l'organisation demande aujourd'hui que cette expérience (par ailleurs toujours nécessaire) soit formalisée par des formations universitaires. Nous avons d'ailleurs observé qu'un nombre non négligeable d'employés du siège suivent parallèlement une formation (financée parfois en partie par l'organisation) afin de capitaliser leurs savoirs pratiques. Cependant, contrairement à la plupart des entreprises classiques, il n'est pas tenu compte des diplômes obtenus dans le calcul de la rémunération.

\subsection{La transmission de l'information, entre canaux formels et informels}

Nous avons observé différents modes de communication dans l'organisation. Certains se sont fortement développés au cours des dernières années. Ainsi, les échanges de courriels sont très nombreux. De nombreux collaborateurs relèvent l'impossibilité de tous les lire et effectuent un tri suivant différentes stratégies. Le nombre de séances auxquelles ils sont tenus d'assister est également perçu comme trop élevé. Là aussi, nous avons observé des résistances importantes : les séances commencent très souvent avec du retard (les participants n'arrivent pas à l'heure) et durent plus longtemps que prévu. Elles n'ont en général pas d'ordre du jour ou alors celui-ci est peu précis et soumis à de nombreux imprévus.

Parallèlement à ces systèmes formels, nous avons observé qu'une importante partie de l'information s'échange par des canaux informels. Les réseaux d'amis sont souvent utilisés afin d'obtenir des services plus rapidement, avec pour conséquence de courtcircuiter les processus hiérarchiques établis. Les rencontres après le travail au bar voisin et les quelques mètres carrés devant les portes servant aux pauses cigarettes sont aussi des lieux d'échanges très fréquentés. Ceci conduit certaines personnes à mettre en place des stratégies pour accéder à ces informations. Les fumeurs d'un service se voient ainsi "délégués » par leurs collègues pour la collecte d'informations auprès de personnes peu disponibles par ailleurs, ou encore certaines personnes effectuent des «rondes» dans le bâtiment afin de se faire voir et de générer des échanges d'informations nécessaires à leur travail, mais que leurs collègues n'auraient peut-être pas pensé à leur transmettre s'ils ne les avaient rencontrés "fortuitement ». Ces types d'échanges sont très valorisés par les employés, au point que les personnes qui ne participent pas à cette « vie sociale » hors du travail (apéros, activités le week-end, etc.), le plus souvent pour des raisons familiales ou de nécessité de coupure, disent s'exclure d'une partie de la vie de l'organisation et manquer ainsi des informations importantes.

Interviewé: "Une partie de [la vie de notre organisation], c'est dans ces moments informels hors de [l'organisation]. Et donc en choisissant de ne pas le faire je choisis de ne plus avoir 
accès entre guillemets à un certain mode de gestion. [...] Je ne maximalise pas les capacités de pouvoir être cent pour cent [pour mon organisation] comme Monsieur et Madame tout le monde [ici]. [...]

Intervieweur: Vous dites qu'il y a beaucoup de choses qui se passent dans ces moments informels, mais c'est quoi, c'est justement quand on invite des collègues chez soi ou

Interviewé: Oui tout à fait, enfin ou le boulot est fini on se retrouve au café du coin, [...] on parle d'un tel, d'une telle, et à ce moment-là s'échange non seulement une relation qui dans le boulot pourra potentiellement faciliter ou non la relation professionnelle, et puis on parle de choses qui ont trait au travail. Et donc ce moment de partage de l'information ou de discussion sur quelque chose de purement professionnel, il n'est plus nécessaire de le refaire au moment du travail. Et donc ceux qui n'y ont pas participé directement ou indirectement... ben voilà. » (19/14/26) (12/21/17)

\subsection{L'aménagement des espaces de travail} communautaire et suscitent une identification forte. Réunies autour d'une cause qui leur est commune et habitées d'un idéal d'engagement personnel, les personnes développent de forts sentiments d'attachement à l'organisation. Nous avons ainsi observé qu'une partie très importante de la vie des employés est axée autour de l'organisation, également dans la sphère hors travail. Avant d'être engagés au siège, la majorité des employés ont connu une expatriation de plusieurs années et beaucoup se sont éloignés de leur région d'origine pour venir au siège. La distance avec leurs cercles d'amis et avec leur famille, ainsi que le mode de vie « en communauté » qu'ils ont connu sur le terrain les ont ainsi conduits à construire une part importante de leur vie sociale autour de l'organisation. Bien que de nombreuses personnes mentionnent la nécessité de développer leur sphère personnelle en-dehors de l'organisation afin de maintenir un équilibre entre vie professionnelle et vie privée, cette dernière se construit aussi bien souvent avec des personnes de l'organisation :

Intervieweur: " Tu as dit qu'il y a beaucoup de la vie sociale qui se fait entre [personnes de

l'organisation], c'est encore le cas maintenant? 
Interviewé: Oui, c'est encore beaucoup le cas, mais voilà on sait quand même, ma femme et moi puisqu'elle est aussi en plein dans le truc, on sait s'en préserver. [...] Une partie de notre temps est axée autour de ça, mais après on s'est construit volontairement une vie en dehors, un truc qui est très protégé, très axé sur nous, [...] d'autres centres d'intérêts [...] qui sont endehors. Mais encore une fois c'est... tout [s'imbrique] puisque les gens qui participent à cette vie-là, [...] qui font exister notre vie à l'extérieur de [l'organisation] sont aussi des gens qui viennent de [l'organisation]. » (21/6/34)

interindividuelles en formalisant les rapports de pouvoir. Les conflits, la concurrence entre collègues, les sentiments d'être exclu ou désigné comme bouc-émissaire ne sont pas absents de cet environnement. On est loin du monde associatif idéal parfois fantasmé. Certaines personnes ont décrit des environnements et des ambiances de travail très négatifs. Une personne décrit par exemple l'ambiance avec ses collègues à la suite d'un événement particulier dans son service :

Interviewé: «J'ai besoin d'avoir un environnement positif et supporteur pour pouvoir travailler. Arriver et que les gens fassent la gueule, ne t'arrosent plus les plantes et te bypass juste parce qu'ils n'arrivent même plus à te demander un truc parce que le climat est tellement tendu, moi je ne peux pas. J'ai déjà beaucoup de boulot, si en plus il faut se battre contre la tronche du voisin... [...] ça moi je ne peux pas. » (13/8/13)

La nature «fonctionnelle » des relations entre collègues s'exprime également dans le fait qu'elles soient déterminées dans le temps. Malgré la très forte composante amicale de ces relations, plusieurs personnes en ont relevé la non-persistance au-delà de la durée d'engagement :

Interviewé: «C'est assez bizarre, on a l'impression de faire partie d'une famille et une fois que les gens partent ils n'existent plus et on ne vous reconnaît même pas dans la rue.» $(17 / 7 / 11)$

Interviewé: "Ma collègue est partie il y a quinze jours, elle est "morte ». Il n'y a pas de... tant que tu donnes le service tu es là, mais tu ne restes pas dans l'imaginaire des gens, dans l'histoire, dans la mémoire des gens. » (13/11/30) 
(emble donc qu'une fois que les personnes quittent l'organisation, elles disparaissent le plus souvent de la vie de l'organisation. Nous pouvons donc aisément imaginer les difficultés alors rencontrées lorsqu'une part si importante de la vie, non seulement professionnelle mais également personnelle, se trouve soudainement amputée.

Les résultats principaux sont résumés dans le tableau 2. Les éléments que nous avons présentés peuvent être organisés selon qu'ils relèvent d'éléments liés au fonctionnement associatif et militant à l'origine de l'organisation (logique associative), ou d'aspects de son fonctionnement liés à sa professionnalisation ou à son institutionnalisation progressive (logique institutionnelle). Ce tableau propose une vue comparée de ces différents éléments.

Tableau 2. Récapitulatif des éléments témoignant des deux logiques, associative et institutionnelle, présentes dans l'organisation

\begin{tabular}{|c|c|c|}
\hline & $\begin{array}{l}\text { Éléments relevant d'une logique } \\
\text { associative }\end{array}$ & $\begin{array}{l}\text { Éléments relevant d'une logique } \\
\text { institutionnelle }\end{array}$ \\
\hline \multirow{3}{*}{ Pouvoir } & Salariés membres de l'association & $\begin{array}{l}\text { Séparation entre les entités } \\
\text { décisionnelles et exécutives }\end{array}$ \\
\hline & $\begin{array}{l}\text { Culture du débat, devoir de donner } \\
\text { son avis, valorisation de la «force de } \\
\text { proposition» }\end{array}$ & $\begin{array}{l}\text { Pouvoir de décision appartenant aux } \\
\text { acteurs opérationnels }\end{array}$ \\
\hline & $\begin{array}{l}\text { Activation des réseaux } \\
\text { indépendamment } \\
\begin{array}{l}\text { des } \\
\text { hiérarchiques }\end{array}\end{array}$ & «Verticalisation » de la hiérarchie \\
\hline Processus & $\begin{array}{l}\text { Règles pas toujours appliquées, } \\
\text { sentiment qu'elles ne sont pas } \\
\text { nécessaires }\end{array}$ & $\begin{array}{l}\text { Efforts de clarification des rôles et des } \\
\text { tâches (cahier des charges, } \\
\text { évaluations annuelles, règlements) }\end{array}$ \\
\hline Communication & $\begin{array}{l}\text { Valorisation des canaux informels } \\
\text { (rencontres après le travail, pauses } \\
\text { cigarettes, tournées) } \\
\text { Retard aux séances, courriels pas } \\
\text { tous lus }\end{array}$ & $\begin{array}{l}\text { Renforcement des modes de } \\
\text { communication formels (séances, } \\
\text { courriels, rapports) }\end{array}$ \\
\hline Formation & $\begin{array}{l}\text { Valorisation de l'expérience du } \\
\text { terrain et de l'« opérationnalité » }\end{array}$ & $\begin{array}{l}\text { Demande de validation des acquis par } \\
\text { des formations universitaires }\end{array}$ \\
\hline $\begin{array}{l}\text { Espaces } \\
\text { travail }\end{array}$ & $\begin{array}{l}\text { Espaces favorisant les échanges et } \\
\text { les rencontres }\end{array}$ & $\begin{array}{l}\text { Cloisonnement des espaces, besoin de } \\
\text { s'isoler }\end{array}$ \\
\hline Relationnel & $\begin{array}{l}\text { Importante vie sociale hors travail, } \\
\text { relations amicales, tutoiement }\end{array}$ & $\begin{array}{l}\text { Relations professionnelles parfois } \\
\text { empreintes de concurrence et de } \\
\text { conflits }\end{array}$ \\
\hline
\end{tabular}




\section{Discussion}

logique institutionnelle dans un environnement auparavant caractérisé par une logique associative. D'une manière générale, cette évolution a permis une plus grande transparence financière et une meilleure reconnaissance du statut professionnel des travailleurs humanitaires. Cependant, notre analyse a montré, en ce qui concerne l'ONG spécifiquement étudiée ici, que la logique institutionnelle n'est pas venue remplacer la logique associative. Nous avons remarqué dans l'activité la cohabitation d'éléments relevant de ces deux logiques, a priori opposables dans leurs modes de fonctionnement.

Nos résultats sont en ligne avec la littérature sur la professionnalisation des ONG. L'importante complexité de ce mouvement et les tensions qui en découlent ont été mises en évidence par plusieurs auteurs (Quéinnec, 2003; Quéinnec, 2007; Dauvin, 2004; Brauman, 1996; Roberts et coll., 2005). Le Naëlou (2004, p. 797) relève les contradictions introduites par la professionnalisation, celle-ci étant "porteuse de spécification, de hiérarchie, de division, alors que beaucoup de projets d'ONG se fondent sur une relative unité et reposent sur une forme d'égalité ». La complexité de la situation de gouvernance a également été relevée par Quéinnec et Haddad (2004, p. 203), qui mettent en évidence une structure du pouvoir relativement confuse au sein des ONG orientées sur des projets opérationnels, et remarquent que la dichotomie entre associatif et exécutif y est plus formelle que réelle.

51 Cette cohabitation peut être analysée avec l'aide du modèle des configurations structurelles proposé par Mintzberg (1978). Les éléments que nous avons présentés peuvent être rapprochés de deux types de structures : la structure entrepreneuriale et la bureaucratie professionnelle. Quéinnec $(2004$, p. 83) qualifie les associations du type de celle présentée ici, à leurs débuts, de "configuration entrepreneuriale localement matinée d'adhocratie ». Cette analyse est pertinente pour l'organisation que nous avons étudiée, ainsi que l'indiquent, à son origine, la simplicité de sa structure, l'absence de différenciation entre ses unités, sa faible hiérarchisation et son fonctionnement très informel (Mintzberg, 1978, p. 375). Nous considérons que cette organisation s'est ensuite développée vers une forme plus proche d'une bureaucratie professionnelle, sous la pression d'une croissance importante et d'un renforcement du contrôle externe. En lien avec la description de Mintzberg, nous avons observé une organisation qui s'appuie sur les compétences de ses employés, qui sont des professionnels dans leur domaine et qui profitent d'une autonomie (relativement) importante. À noter que le terme de bureaucratie est ici employé dans son sens technique se référant à la standardisation des comportements de l'organisation et non dans une appréciation péjorative liée à la rigidité des actions (Mintzberg, 1978, p. 102). Dans le mouvement de professionnalisation qu'a connu l'ONG étudiée, la standardisation s'est vu introduite principalement dans les procédés de travail, comme nous l'avons décrit sous le point 3.2 des résultats sur la codification du travail, ainsi que dans les qualifications des employés, avec notamment la valorisation croissante des formations spécialisées dans l'humanitaire.

Cependant, un nombre important d'éléments relevant d'une structure entrepreneuriale persiste au sein de la forme plus bureaucratique progressivement mise en place. Ces deux formes cohabitent au sein de l'organisation, ce qui conduit à un mode de fonctionnement « hybride» (Mintzberg, 1978, p. 413). Le siège s'est vu de plus en plus structuré sur un 
modèle bureaucratique (ligne hiérarchique claire, standardisation du travail, formalisation des modes de communication, etc.) s'éloignant du modèle associatif, ou entrepreneurial, original ; néanmoins la « culture organisationnelle » continue à valoriser le mode de fonctionnement associatif (culture du débat, valorisation des canaux de communication informels, importance de la vie sociale hors travail, etc.). Le travail s'est ainsi vu codifié, cependant, les nombreuses résistances faites à l'application des règles formelles et des procédures standardisées (et le fait qu'elles ne soient pour la plupart pas imposées trop strictement) contribuent à maintenir l'illusion d'une structure où s'exerce très peu de contrôle.

De nombreux points de tensions dans les formes d'organisation du travail résultent de cette cohabitation (voir tableau 2). Cela rend difficile la perception des limites de ce qui est acceptable ainsi que l'appréciation de la marge de manœuvre pour chaque employé. De forts sentiments d'inégalité de traitement ainsi qu'une importante insatisfaction, source de mal-être au travail, ont été rapportés en lien avec ces éléments. Nos analyses montrent que cette situation d'hybride résulte de la nécessité perçue de ne pas trancher trop nettement pour une position afin de profiter des avantages des deux formes organisationnelles. Dauvin et Siméant (2002, p. 120) relèvent ainsi : "[...] salariés et bailleurs (de fonds) en arrivent à promouvoir de façon convergente une conception de l'humanitaire qui se traduit par une professionnalisation croissante, assez floue cependant pour qu'elle soit présentable - et vivable - sur un registre militant de la part des salariés des ONG. " Les ONG se retrouvent donc dans une situation particulière puisqu'elles sont dans l'obligation de se professionnaliser pour rester compétitives, mais qu'elles doivent également maintenir une image militante et associative forte pour entretenir et valoriser l'engagement de leurs membres. Pour le cas présenté ici, cette double injonction empêche l'achèvement de la transition d'une forme vers une autre. Si l'ONG semble avoir besoin de faire cohabiter structure associative et structure institutionnelle au sein de son siège pour fonctionner, cette cohabitation ne se fait pas pour autant sans heurts. Elle crée une dynamique contradictoire et génère des sentiments d'instabilité et d'inégalité. Il semble donc bien qu'elle ait un « coût " pour les employés. En introduisant les termes d'efficacité, de rentabilité et de compétences là où les mots de solidarité, de militance et d'engagement dominent (Le Naëlou, 2004, p.776-7), les méthodes managériales appliquées dans les ONG vont souvent heurter les valeurs à l'origine de l'engagement (Dauvin, 2004, p. 833). L'atteinte à ces valeurs, fondamentales dans le milieu humanitaire, risque de conduire à une perte de sens du travail, laquelle est une cause maintenant établie de souffrance au travail (Davezies, 1999, p. 103 ; Dejours, 2000 ; Morin, 2006).

$\mathrm{Au}$ delà des sentiments d'instabilité et d'inégalité générés, nous pensons que cette situation d'«hybride" a des effets sur l'économie des échanges et du lien dans l'organisation. La littérature sur le don et le contre-don offre sur ce point un angle d'analyse pertinent. Mauss (2007, parution originale en 1925) a montré la nécessité du contre-don pour maintenir le lien social, et l'importance des échanges par le don dans le monde du travail a depuis été mise en évidence par plusieurs auteurs (Gonik, 2006 ; Alter, 2008 ; Alter, 2010 ; Chanial, 2008). Dans le contexte étudié, la part du don des salariés représente un enjeu central de leur engagement. Ces derniers renoncent à certains avantages économiques (rémunération inférieure au niveau du "marché ») et acceptent des contraintes importantes (longues heures de travail, réunions le week-end, etc.) au nom de la cause qu'ils servent (Alter, 2010, p. 126 ; Crochet, 2008, p. 390). La logique du 
sacrifice est nécessaire au fonctionnement de l'organisation. Cependant, elle s'accorde mal avec la logique du management, ce dernier ne peut y répondre sur le même registre. Il en résulte une non-reconnaissance du don: "Refuser ce don permet de ne pas le célébrer, [...] de ne pas avoir à rendre compte de ce qui est fait et, surtout, de ne pas en être redevable.» (Alter, 2010, p. 141). L'analyse sous cet angle permet de mettre en évidence les contradictions générées par cette situation d'hybride. L'organisation se dédouane de la nécessité du contre-don, elle se "cache » derrière un fonctionnement professionnel en réponse à un don de l'ordre associatif et militant. Les compensations attendues par les employés, qui sont de l'ordre de la participation associative et militante, ne trouvent plus écho dans un modèle professionnalisé. La situation est complexe puisque le discours de la vocation et de l'engagement sans attente de retour est soigneusement entretenu dans l'organisation, aussi par les discours managériaux. Le message est donc hautement contradictoire : l'organisation demande à ses employés d'être engagés comme envers une famille, mais les règles sont celles d'une entreprise. Les acteurs sont contraints de jongler entre ces différents registres avec les risques que cela comporte.

En résumé, nos résultats ont permis de mettre en évidence les conséquences de la professionnalisation de la structure sur l'activité et sur l'organisation du travail au sein d'une ONG donnée. Nous avons montré qu'il en résulte une situation dans laquelle exigences managériales et vocation militante cohabitent. Cette situation "hybride " contribue à générer des messages paradoxaux, des questionnements sur le sens du travail et des déséquilibres entre don et contre-don, lesquels produisent une situation potentiellement pathogène. Le climat organisationnel décrit a des effets sur la santé des collaborateurs et n'est pas étranger aux problématiques de mal-être au travail observées par ailleurs dans cet environnement.

Une des limites inhérente au modèle de l'étude de cas adopté ici est le fait que cette étude ne porte que sur une seule ONG. La situation observée et décrite ici n'est donc pas généralisable à l'ensemble des organisations de solidarité internationale. De plus, notre analyse s'est limitée à une composante de cette ONG, à savoir son siège. Ces choix méthodologiques nous ont néanmoins permis de réaliser une analyse fine et approfondie. De plus, notre recherche représente une observation à un temps T. C'est notre connaissance de l'histoire de l'organisation qui nous permet de relier la coexistence des deux formes structurelles observées à son évolution temporelle.

\section{Conclusion}

L'intérêt de ce travail réside dans le fait qu'en partant de l'observation du travail réel des employés, et en enrichissant ces observations de données textuelles et discursives nous informant des prescriptions courant dans l'organisation, nous avons mis en évidence un imbroglio compliqué dont le présent article représente une tentative de "démêlage ». À partir des décalages observés entre travail réel et travail prescrit, cette démarche nous a permis de déceler l'évolution de la structure et de ses modes de fonctionnement. Cet article présente ainsi une étude de cas d'une nouvelle forme d'organisation du travail qui ouvre de nouvelles perspectives pour l'étude du travail, au-delà de la dichotomie traditionnelle entre «association » et « entreprise ». Simonet (2006, p. 205) a montré que ces catégories se « contaminent » entre elles, que chacune emprunte au modèle de l'autre. De plus en plus d'organisations sont amenées à faire cohabiter différents modèles de fonctionnement pour répondre aux contraintes du monde du travail actuel. Notre analyse 
en est une illustration. Nous avons également montré les limites de cet hybride pour le cas particulier étudié ici:tenter de le maintenir conduit à développer des fonctionnements cachés et génère des injonctions paradoxales qui ne sont pas sans effets, à long terme, sur la santé des individus. Jouer sur ces deux tableaux n'est donc pas chose aisée et représente un réel défi pour l'organisation du travail dans son ensemble.

Les auteures remercient les participants à cette étude, ainsi que les évaluateurs pour la qualité de leurs commentaires et leur contribution à l'amélioration de la qualité de cet article.

\section{BIBLIOGRAPHIE}

Alter, N. (2008). Travail, organisation et échange social. In P. Chanial (Ed.), La société vue du don: manuel de sociologie anti-utilitariste appliquée (p. 45-69). Paris : Éditions La Découverte.

Alter, N. (2010). Travail et déni du don. Revue du M.A.U.S.S., 125-142.

Amiel, R. (1986). La notion de santé mentale et son évaluation dans les études épidémiologiques à visées préventives en médecine du travail et en santé communautaire. Archives des maladies professionnelles de médecine du travail et de sécurité sociale, 47, 1-14.

Apostolidis, T. (2006). Représentations sociales et triangulation : une application en psychologie sociale de la santé. Psicologia : Teoria e Pesquisa, 22, 211-226.

Boitel, L., Julliard, G., Blondet, M., Leroux, C., Pardon, N., Viot, P. et coll. (1985). Recherches portant sur les relations travail - environnement et santé des travailleurs. Paris : Publications A.S.M.T.

Brauman, R. (1996). Humanitaire le dilemme. Paris : Éditions textuel.

Brisson, C., Blanchette, C., Guimont, C., Dion, G., Moisan, J., Vézina, M. et coll. (1998). Reliability and validity of the French version of the 18 -items Karasek Job Content Questionnaire. Work \& Stress, 12, 322-336.

Cahill, K. M. (2006). Au fondement de l'action humanitaire. Paris : NiL éditions.

Center for international humanitarian cooperation. (2011). www.cihc.org/. 3-3-2011.

Chanial, P. (2008). La société vue du don : manuel de sociologie anti-utilitariste appliquée. Paris :

Éditions La Découverte.

Crochet, S. (2008). Le sacrifice impossible : contradictions de l'action humanitaire. In P. Chanial (Ed.), La société vue du don : manuel de sociologie anti-utilitariste appliquée (p. 381-398). Paris : Éditions La Découverte.

Dauvin, P. (2004). Être un professionnel de l'humanitaire ou comment composer avec le cadre imposé. Revue Tiers Monde, 180, 825-840.

Dauvin, P., Siméant, J. et C.A.H.I.E.R. (2002). Le travail humanitaire : les acteurs des ONG, du siège au terrain. Paris : Presses de Sciences Po.

Davezies, P. (1999). Évolution des organisations du travail et atteintes à la santé. Travailler, 3, 87-114. 
Dejours, C. (2000). Travail, usure mentale. Paris : Bayard.

Denzin, N. K. (1978). The research act : A theoretical introduction to sociological methods. New York : McGraw-Hill.

Gonik, V. (2006). Le collectif au travail et la santé analysés au travers de la théorie du don. In Ergonomie et santé au travail : Transformations du travail et perspectives pluridisciplinaires. Actes du $41^{\mathrm{e}}$ Congrès de la Société d'ergonomie de langue française.

Jick, T. D. (1979). Mixing qualitative and quatitative methods : Triangulation in action. Administrative Science Quarterly, 24, 602-611.

Karasek, R., Brisson, C., Kawakami, N., Houtman, I., Bongers, P. et Amick, B. (1998). The Job Content Questionnaire (JCQ) : An instrument for internationally comparative assessments of psychosocial job characteristics. Journal of Occupational Health Psychology, 3, 322-355.

Krieger, R. et Graf, M. (2009). Travail et santé: récapitulation des résultats de l'enquête suisse sur la santé 2007 (Rep. No. 2008-09-02/47 COO.2101.104.5.1590646). Zürich.

Le Naëlou, A. (2004). Pour comprendre la professionnalisation dans les ONG : quelques apports d'une sociologue des professions. Revue Tiers Monde, 180, 772-798.

Mauss, M. (2007). Essai sur le don. Paris.

Mintzberg, H. (1978). Structure et dynamique des organisations. Paris : Eyrolles Éditions d'organisation.

Mirvis, P. H. et Hackett, E. J. (1983). Work and Work Force Characteristics in the Nonprofit Sector. Monthly Labor Review, 106, 3-12.

Morin, E. M. (2006). Donner un sens au travail.

Niedhammer, I., Siegrist, J., Landre, M. F., Goldberg, M. et Leclerc, A. (2000). Étude des qualités psychométriques de la version française du modèle déséquilibre efforts/récompenses. Revue d'épidémiologie et de santé publique, 48, 419-437.

Programme interdisciniplinaire en action humanitaire. (2011). www.unige.ch/formcont/piah/ piah.html. 3-3-2011.

Quéinnec, E. (2003). L'ambivalence être/objet des organisations humanitaires : un objet de recherche pour les sciences de gestion. Revue internationale des sciences sociales, 177, 557-580.

Quéinnec, E. (2004). Les grandes ONG humanitaires ou l'exemple original d'un management par le contrôle de soi. In E.Quéinnec et J. Igalens (Eds.), Les organisations non gouvernementales et le management (Vuibert ed., p. 49-89). Paris : Vuibert.

Quéinnec, E. (2007). La croissance des ONG humanitaires. Une innovation devenue institution. Revue française de gestion, 177, 83-94.

Quéinnec, E. et Haddad, L. (2004). Professionnaliser la gestion, gérer le professionnalisme. Les ONG confrontées aux dilemmes de leur croissance. In E. Quéinnec et J. Igalens (Eds.), Les organisations non gouvernementales et le management (Vuibert ed., p. 167-212). Paris : Vuibert.

Roberts, S. M., Jones III, J. P. et Fröhling, O. (2005). NGOs and the globalization of managerialism : A research framework. World Development, 33, 1845-1864.

Rochat, L., Gonik, V. et Danuser, B. (2010). Travailler dans l'urgence au quotidien : quels impacts sur les employés et sur l'organisation ? Étude qualitative au siège d'une ONG humanitaire. Journal international de santé au travail, 1-10. 
Simonet, M. (2006). Le monde associatif : entre travail et engagement. In N. Alter (Éd.), Sociologie du monde du travail (p. 191-207). Paris : Presses universitaires de France.

Young, D. R. et Billis, D. (1990). Editors' notes. Nonprofit Management and Leadership, 1, 1-5.

\section{NOTES}

1. Pour des raisons de confidentialité, le nom de l'ONG n'est pas cité.

2. Nous utilisons ici le terme "terrain" dans le sens où il est utilisé par les acteurs de l'organisation. Le travail dit « de terrain » regroupe toutes les activités s'effectuant auprès des populations auxquelles s'adresse l'aide prodiguée par cette organisation.

3. Ce questionnaire comprenait pour une part des instruments validés: le «Job Content Questionnaire » de Karasek (Karasek et coll., 1998 ; Brisson et coll., 1998), le «Test de Santé Total » de Langner, traduit et validé par Amiel (Boitel et coll., 1985), ainsi que des items issus de l'échelle de Siegrist (Niedhammer, Siegrist, Landre, Goldberg et Leclerc, 2000). Des questions sur le type de nuisances perçues au poste de travail, sur le stress ressenti ainsi que sur la conciliation entre vie privée et vie professionnelle ont également été posées.

4. Lorsqu'il n'était pas possible (ou pas souhaité) de suivre une personne désignée, c'est une équipe ou un lieu qui a fait l'objet de l'observation.

5. Les termes d'«opérations" et d'«opérationnel » sont ici utilisés pour rendre compte des fonctions et des activités les plus directement en lien avec les terrains d'intervention.

\section{RÉSUMÉS}

Le milieu de l'humanitaire a connu des évolutions importantes durant les dernières décennies, impliquant des transformations dans les modes de fonctionnement des organisations d'aide. Nous avons réalisé une recherche au siège d'une ONG s'inscrivant dans ce contexte. Par le biais d'analyses de l'activité, d'entretiens et d'un questionnaire, nous avons mis en évidence les éléments introduits dans le contexte de la professionnalisation de la structure de cette organisation et leur perception par les acteurs. Nous avons observé la persistance de nombreux éléments relevant de la logique associative au sein de ce qui tend à devenir une institution. Les décalages résultant de la cohabitation de ces deux logiques de fonctionnement, associative et institutionnelle, créent un mode d'organisation du travail particulier et génèrent des messages paradoxaux pour les employés, lesquels peuvent avoir des effets sur leur santé.

In recent decades, the context of humanitarian aid has undergone many developments involving changes in the means of operation of aid organizations. We conducted a study at the headquarters of an NGO, in which we collected data via a questionnaire, semi-structured interviews and activity analyses. We identified the elements implemented in the process of the professionalization of the management structure and how they were perceived by the employees. Numerous aspects of the associative ideal remain strong in the organization. Resistance against formal management exists, which leads to the cohabitation of these two ways of functioning which we called associative and institutional. Shifts resulting from this 
cohabitation create a particular mode of organization and generate paradoxical messages for the employees, which may have impacts on their health.

En las últimas décadas, el contexto de la ayuda humanitaria ha conocido evoluciones importantes, lo que provocó transformaciones en el funcionamiento de las organizaciones de ayuda. Realizamos una investigación en la sede de una ONG que se inscribe en este contexto. Los elementos introducidos en el proceso de profesionalización de su estructura y la percepción que los protagonistas tienen de ella, se pusieron en evidencia por medio del análisis de la actividad, de entrevistas y de un cuestionario. Observamos la persistencia de numerosos elementos relacionados con la lógica asociativa en el seno de este organismo que tiende a devenir una institución. Los desfases que resultan de la cohabitación de las dos lógicas de funcionamiento : asociativa e institucional, crean un modo de organización de trabajo particular y generan mensajes paradójicos para los empleados, los cuales pueden tener efectos en su salud.

\section{INDEX}

Mots-clés : professionnalisation, organisation non gouvernementale, humanitaire, santé mentale, travail

Keywords : professionalization, nongovernmental organization, humanitarian, occupational health, managerialism, nonprofit sector

Palabras claves : profesionalización, organización no gubernamental, humanitarian, salud mental, trabajo

\section{AUTEURS}

\section{LYSIANNE ROCHAT}

lysiane.rochat@hospvd.ch, Institut universitaire romand de santé au travail (IST) - Rue du Bugnon 21, 1011 Lausanne - Suisse

\section{VIVIANE GONIK}

viviane.gonik@hospvd.ch, Institut universitaire romand de santé au travail (IST) - Rue du Bugnon 21, 1011 Lausanne - Suisse

\section{BRIGITTA DANUSER}

brigitta.danuser@hospvd.ch, Institut universitaire romand de santé au travail (IST) - Rue du Bugnon 21, 1011 Lausanne - Suisse 